\title{
A Multistage Technique to Minimize Overestimations of Slope Susceptibility at Large Spatial Scales
}

\author{
Avalon-Cullen $\mathbf{C}^{1^{*}}$, Kashuk $\mathbf{S}^{2}$, Suhili $\mathbf{R}^{2}$, Khanbilvardi $\mathbf{R}^{2}$ and Temimi $\mathbf{M}^{2,3}$ \\ ${ }^{1}$ Department of Earth and Atmospheric Science, Graduate Center City, University of New York, USA \\ ${ }^{2}$ Department of Earth and Atmospheric Sciences and NOAA-CREST center, The City College of New York, USA
}

${ }^{3}$ Water Center, Masdar Institute of Science and Technology, Abu Dhabi, United Arab Emirates

*Corresponding author: Avalon-Cullen C, Department of Earth and Atmospheric Science, Graduate Center City, University of New York, USA, Tel: 212-491-9118; Email: ccullen@gradcenter.cuny.edu/skashuk@ccny.cuny.edu

Received date: Feb 22, 2016; Accepted date: Mar 03, 2016; Published date: Mar 8, 2016

Copyright: ( 2016 Cullen CA, et al. This is an open-access article distributed under the terms of the Creative Commons Attribution License, which permits unrestricted use, distribution, and reproduction in any medium, provided the original author and source are credited.

\begin{abstract}
Rainfall induced landslides are one of the most frequent natural hazards on slanted terrains. They lead to significant economic losses and fatalities worldwide. Most factors inducing shallow landslides are local and can only be mapped with high levels of uncertainty at larger scales. This work presents an attempt to determine slope instability using buffer and threshold techniques to downscale large areas and minimize slope uncertainties at local scales, then in a second stage, logistic regression is used to determine susceptibility at large scales. ASTER GDEM V2 is used for topographical characterization of slope and buffer analysis. Four static parameters (slope angle, soil type, land cover and elevation) for 230 shallow rainfall-induced landslides listed in a comprehensive landslide inventory for the continental United States are examined. A delimiting buffer equivalent to 5,25 or $50 \mathrm{~km}$ is created around each landslide event facilitating the statistical analysis of slope thresholds. Slope angle thresholds at the pixel points 50, 75, 95, 99 and maximum percentiles are compared to one another and tested for best fit in a logistic regression environment. It is determined that values lower than the 75-percentile threshold misrepresents susceptible slope angles by not including slopes higher than $35^{\circ}$. Best range of slope angles and regression fit can be achieved when utilizing the 99 percentile slope angle threshold. The resulting logistic regression model predicts the highest number of cases correctly with $97.2 \%$ accuracy. The logistic regression model is carried over to ArcGIS where all variables are processed based on their corresponding coefficients. A regional landslide probability map for the continental United States is created and analyzed against the available landslide records and their spatial distributions. It is expected that future inclusion of dynamic parameters like precipitation and other proxies like soil moisture into the model will further improve accuracy.
\end{abstract}

Keywords: Shallow landslides; Slope instability; Threshold analysis; Logistic regression; Regional analysis; GIS; Remote sensing

\section{Introduction}

Rainfall induced landslides are one of the most frequent natural hazards on slanted terrains. They usually result in great economic losses and fatalities globally. Worldwide at least 32,322 deaths between 2004 and 2010 have been reported [1] and in the United States alone, landslides cause \$1-2 billion in damages and more than 25 fatalities in average each year [2]. Understanding, mapping, modeling and preventing the aftermath of these devastating events represents an important scientific and operational endeavor [3].

The term "Landslide" describes the downward and outward movement of slope-forming materials that include rock, earth, and debris or a combination of these [4]. Although landslides are considered to be dependent on the complex interaction of several static and dynamic factors [5-7] slope angle has great influence on the susceptibility of a slope to sliding. Increased slope angle usually correlates to increased likelihood of failure even if the material distribution on the slope is uniform and isotropic [5]. Undeniably, many other parameters are essential to the analysis of landslide risk. For example, changes in land use and land cover such as deforestation, forest logging, road construction, cultivation and fire on steep slopes can have a significant effect on landslide activity [8]. In addition, forest vegetation, especially tree roots help stabilizes hill slopes by reinforcing soil shear strength. Root reinforcement is imperative on slopes where roots can extend into joints and fractures in bedrock or into a weathered transitional layer between the soil and bedrock $[9,10]$.

Furthermore, soil properties such as particle size and pore distribution of the soil matrix influence slope instability. These properties influence the soil's holding capacity and rate in which water moves through the soil. Coarse soils are known to hold less water under unsaturated conditions than finer soils [11]. Rainfall intensity and duration affect the soil's saturation level. Hence, hydraulic characteristics and matrix suction properties of soil are crucial in the study of rainfall triggered shallow landslides [12]. In general, soil types and their associated geotechnical, mechanical, physical and hydrological properties are essential for the assessment of landslide hazards [8].

Various studies that list, define areas of susceptibility and attempt to forecast landslides have shed some light on the conditions and mechanisms that influence slope instability [12-18]. Nonetheless, the reliability of all proposed methodologies is dependent on the availability of adequate temporal and spatial surface data in addition to adequate reporting [19]. At local scales, deterministic methods are considered to be most reliable because they are founded on geotechnical properties [20]. Nevertheless, deterministic methods are inadequate for the study of landslides at large scales as geotechnical 
and hydrological conditions vary persistently from location to location [20].

Statistically based models are preferred at large scales as they are known to have a good degree of reliability correlating instability parameters to past distribution of landslides [14]. Logistic regression, for example, is one of the most common statistical methods used for landslide assessments [12,17,21-25]. Logistic regression is used to find the best fitting relationship of multiple independent variables to a dependent variable and does not require normally distributed landslide conditioning parameters. Logistic methods are a multivariable analysis technique where the dependent variable is not a continuous parameter and where the result is a binary probability of values between 0 and 1 [22]. The advantage of logistic methods over regression analysis and discriminant analysis for the study of landslides is the fact that the dependent variable has the probability of only two values: an event happening or not happening ( 0 or 1$)$ [26,27].

The advancement of remote sensing techniques offers a better opportunity to analyze landslide risk at large scales; however, great discrepancies arise when monitoring landslides at high spatial resolution over a large domain. Inventories usually depend on information retrieved from newspapers, online news, and government agencies where heterogeneous reporting is unavoidable. In many instances, catalogs lack precise spatial and temporal distribution making it hard to identify the precise conditions involved in the development of landslide events. In addition, studies have shown that susceptible slope angle is misrepresented at large scales. Kirschbaum [6] for example, emphasizes that slope angle values in Hong [5] global model are under-valued at around $21^{\circ}$ due to averaging values over a large area. Similarly, in a global landslide hotspot study, Nadim [26] places susceptible slope angle between 8 and $32^{\circ}$ top. Defining a better technique than just finding the average can assist to reduce slope under-estimations at large-scales and be very helpful for the analysis of landslide risks.

This work proposes to address the scale dilemma by utilizing a descriptive landslide inventory in addition to buffer and threshold techniques that help minimize susceptibility overestimation at large spatial scales. Precisely, the proposed blended techniques involve a reducing the area of study as a suitable approach that delineates areas of high risk where another approach that is appropriate for large assessment is applied. It is then a multistage approach that is proposed here to bridge the gap between different appraisal scales and reduce slope misrepresentations. These two techniques are applied in the spatial context of the Continental United States utilizing the best available rainfall-triggered landslide inventory that represents most of the dominant conditions of landslide prone localities. Subsequently, logistic analysis is used to determine landslide probability at the regional scale.

\section{Methods}

This work presents a multistage technique that bridges the gap between landslide mapping at large and local scales. Based on a descriptive (spatial and temporal) landslide record, buffers are used to condense the area of study to that of the most likely area of slope susceptibility. Consequently, various percentile thresholds for each static parameter are tested in a logistic regression model to determine the best fit. Validation of the model is performed by the random division of the data in a 70-30\% fashion and data partition and crossvalidation. A confusion matrix helps conclude details about the performance of the model. Best fitting model is then represented in a landslide probability map for the continental United States.

\section{Data collection}

Advanced Spaceborne Thermal Emission and Reflection Radiometer (ASTER) Global Digital Elevation Model (GDEM) 10 by 10 tiles were merged into a single DEM utilizing ArcGIS's mosaic to new raster function. Soil type was obtained from the Harmonize World Soil Database Version (HWSD) 1.2. This dataset combines existing regional and national updates of soil information from around the world and incorporates them into the Food and Agriculture Organization of the United Nations (FAO-UNESCO) soil Map of the World at a $1 \mathrm{~km}$ resolution. Land cover was retrieved from the FAO Global Land Cover-SHARE database at $1 \mathrm{~km}^{2}$ resolution. This dataset integrates local and global land cover information, local information is derived from datasets such as Africover and Corine LC and global data is derived from the Moderate-resolution Imagine Spectroradiometer MODIS Vegetation Continuous Fields VCF2010 [28,29].

\section{Buffer analysis}

Landslide inventory: Developing an approach for local and regional monitoring of landslides is possible when a large and comprehensive record of landslides events is available, this represents the main limitation of this work. Obtaining event data or a consolidated landslide inventory at large scales is extremely challenging due to heterogeneous reporting and data availability even for a country such as the United States. The United States Geological Survey (USGS) is currently compiling a listing of global and local events [30] but uniform reporting is not available yet. In addition, events listed in the State geological surveys, in many instances, lack precise spatial and temporal distribution.

To this day, the most uniform and comprehensive landslide inventory found by the authors is being developed at the National Aeronautics and Space Administration (NASA) and explained in Kirschbaum [6]. The inventory is a systematic landslide catalog that lists around 1,600 landslides globally and 270 for the United States for the years 2003, 2007, 2008 and 2009. The inventory summarizes rainfall-triggered landslides and debris flows reported in newspapers, online news, and government agencies. Landslide events are reported with an accuracy of 24 hours, and in the case of multiple landslides occurring during one rainfall event, the first landslide is designated as the event time.

This particular inventory stands out from other listings because two qualitative indices were designated to represent locality and size uncertainties that are otherwise kept undefined in other inventories. Index 1: Confidence radius, represents general location accuracy, and Index 2: Size radius, differentiates small from larger events as well as minor events from catastrophic events. Both indices range on a scale between 0 and 5 where 5 represent the most accurate location and the biggest event respectively as seen in Table 1 [6].

In this study, size radius and confidence radius are adopted. Size radius is incorporated as a measure of landslide size and confidence radius as an extent of uncertainty. Only confidence radius of 5, 25 and $50 \mathrm{Km}$ are considered as they represent the exact or near the exact location, a location known to the extent of a city or nearby coordinates of a village respectively. 
Page 3 of 7

\begin{tabular}{|l|l|l|}
\hline Landslide location classification & Radius of Confidence in landslide location & Reporting of landslide location \\
\hline 3 & $25-50 \mathrm{~km}$ & $\begin{array}{l}\text { Cannot locate city or village where event occurred but can identify } \\
\text { geographic coordinates of nearby cited location }\end{array}$ \\
\hline 4 & $5-25 \mathrm{~km}$ & Landslide location known within the extent of city or village \\
\hline 5 & $<5 \mathrm{~km}$ & Exact or near exact location within city or along road \\
\hline Landslide size classification & Relative landslide size & Reporting of landslide size \\
\hline 3 & $\begin{array}{l}\text { Multiple sliding events over a region, medium to large } \\
\text { event affecting a large area or population }\end{array}$ & Multiple events within an area; multiple events along road cuts \\
\hline 4 & $\begin{array}{l}\text { Large to massive landslide, many landslide which } \\
\text { affected one general area }\end{array}$ & Landslide affected an entire village, city, or series of villages \\
\hline 5 & $\begin{array}{l}\text { Massive event or multiple large events, often causing } \\
\text { fatalities }\end{array}$ & Affected an entire region \\
\hline
\end{tabular}

Table 1: Kirschbaum et al. [6] excerpt Landslide inventory confidence and size radius.

Confidence radius greater than $50 \mathrm{Km}$ are described in the inventory as events occurring somewhere within a country or large region making the uncertainty area too large and, therefore, are excluded from the analysis. The resulting 230-landslide events in the US are distributed between Longitudes $60 \mathrm{~W}$ and $130 \mathrm{~W}$ and Latitudes $30 \mathrm{~N}$ and $60 \mathrm{~N}$ resulting in a suitable representation of the locations and characteristics that are known to be prone to landslides in the continental U.S. as per Radbruch-Hall et al. [28]. Buffers equivalent to the extent of the confidence radius are created around each landslide event as seen in Figure 1.

This process helps reduce the area of study to that of the buffer and therefore, it is reasonable to assume that the buffered area includes all possible places in which the event might have occurred. By this means, it is possible to statistically analyze the characteristics of the terrain that could have led to the rain-triggered landslide. Buffer extraction from the original dataset is carried on by an itinerating algorithm that correlates the spatial coordinates of each event to the coordinates in the dataset. Consequently, subsections corresponding to each buffered area are extracted from each dataset and pixel values for each area are converted into ASCII files. Each file corresponds to one buffer or one event, resulting in 230 files for each dataset type.

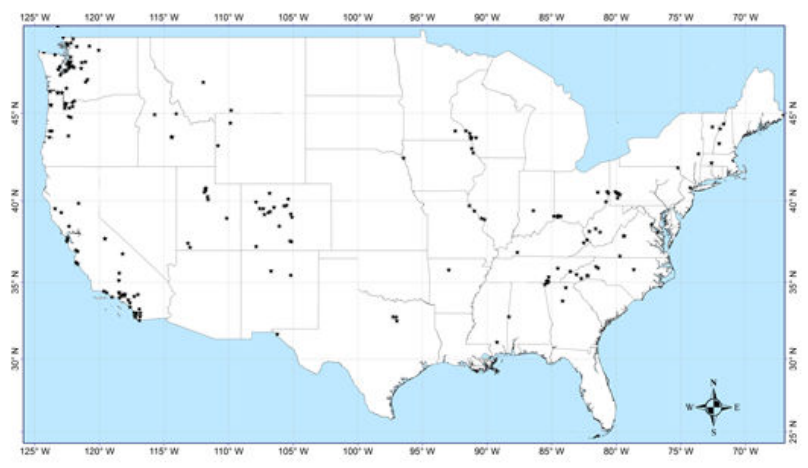

Figure 1: Landslide inventory for 5, 25 and $50 \mathrm{~km}$ confidence radius events in the USA.

\section{Threshold sensitivity analysis}

Slope: Rainfall-induced shallow landslides occur as relatively shallow $(0.3-2 \mathrm{~m})$ failure surfaces parallel to the slope in landslideprone slants [31,32]. In the case of rainfall-induced landslides, slope angle is the underlying factor in downslope movement after gravity forces acting parallel to the slope have superseded friction and cohesion forces. It is undeniably possible that some events could have happened at less steep slopes as gravity alone does not determine downward movement, nevertheless, the likelihood is higher as slope angle increases. This work develops under this premise.

In this work, slope angle values for all 230-landslide events in the continental U.S. are derived utilizing the mosaicked DEM. Pixel values within each buffer are analyzed statistically by creating thresholds, these thresholds are then partitioned and sorted in ascending order. Values are organized in rank order from lowest to the highest, the lowest score is in the $1^{\text {st }}$ percentile and the highest score is the $99^{\text {th }}$ percentile. The percentile represents the value below which a given percentage of the observations lie [33]. For example, if a slope value is in the $99^{\text {th }}$ percentile, it means that it is higher than $99 \%$ of the other slope values.

Percentiles are then used as thresholds in each buffer zone, values laying bellow the specific percentile are considered stable, and values laying above the percentile are considered unstable. Thresholds for the $\mathrm{T}_{\text {point }}=$ Pixel Point, $\mathrm{T}_{50}=50, \mathrm{~T}_{75}=75, \mathrm{~T}_{95}=95, \mathrm{~T}_{99}=99$ and $\mathrm{T}_{100}=\mathrm{Max}$, are tested for all buffers. This technique leads to the assessment of the slope percentiles that result in underestimations and over-estimations. Buffers for 3 landslide events and their corresponding $\mathrm{T}_{99}$ threshold can be seen in Figure 2. 


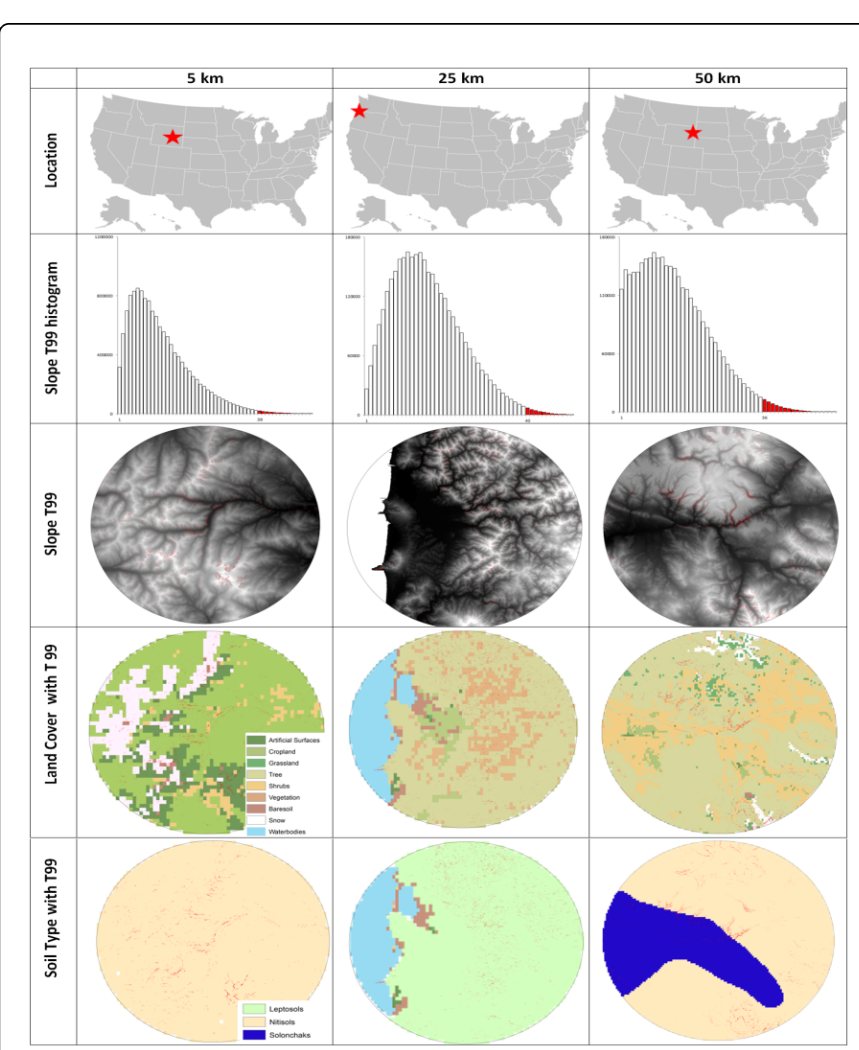

Figure 2: Landslide event buffer and threshold analysis represented for 5,25 and $50 \mathrm{Km}$ for all variables.

Three different events with buffers of 5,25 and $50 \mathrm{~km}$ and their corresponding slope, elevation, land cover and soil type are represented. Percentile threshold $\mathrm{T}_{99}$ is highlighted in red in each histogram as well as in each buffer.

Elevation: Altitude values corresponding to the $\mathrm{T}_{99}$ percentile threshold for all 230-landslide buffers are selected. As with the slope buffers, 230 extractions from the DEM are converted into ASCII files. Analysis of the mean, the standard deviation and other statistical moments is investigated.

Land cover: Land cover classes are represented by numerical values in each data-set; these values are extracted from each buffer and then converted into ASCII files. Because land cover classes are categorical no other statistical moment besides the mode is tested. The corresponding mode for the $\mathrm{T}_{99}$ percentile threshold for each buffer is selected as the prominent land cover value within the buffer.

Soil type: The HWSD lists 36 different soil types and their corresponding physical-chemical properties. Textures, soil drainage, available water storage capacity, soil phase among many other characteristics for each soil are described in the database. Classes found within each buffer are examined and the mode corresponding to the $\mathrm{T}_{99}$ percentage threshold range is selected as the representative value for each buffer as shown in Figure 2.

Threshold values for each file are calculated. A complete flow chart for the analysis framework is illustrated in Figure 3.

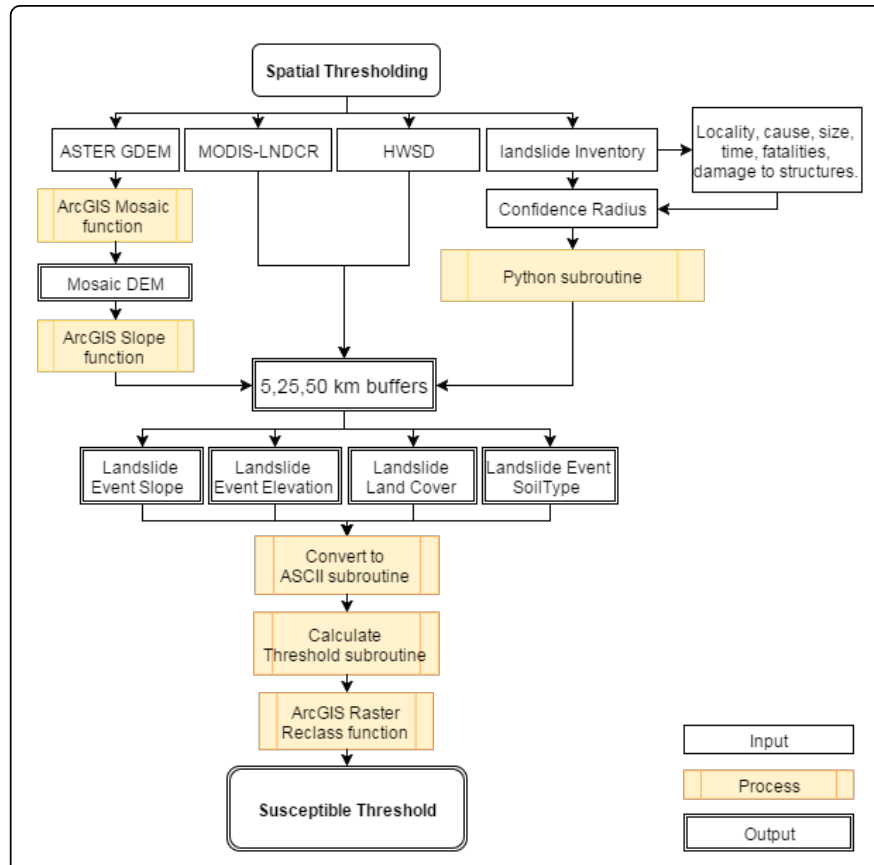

Figure 3: Threshold sensitivity analysis schematic.

\section{Logistic Regression (LR) model}

The LR method is based on the generalized linear model that can be expressed as Probability of Landslide (Pl):

$$
P l=\frac{1}{1+e^{-Z}} \text { Eq.1 }
$$

Where $\mathrm{Pl}$ is the probability of a landslide event expressed in a dichotomous way of 0 and 1 , set by a classification cutoff point value of 0.5 for adjusting the estimated $\mathrm{Pl}$ values to 0 for $\mathrm{Pl}<0.5$ and 1 for $\mathrm{Pl}>=0.5$. The logit $\mathrm{Z}$ is assumed to contain the independent variables on which the landslide event may fall. The $\mathrm{Z}$ term is expressed in the linear form as:

$$
Z=\beta_{0}+\beta_{1} \chi_{1}+\beta_{2} \chi_{2}+\ldots+\beta_{n} \chi_{n} \text { Eq. } 2
$$

Where $\beta_{0}$ represents the intercept of the model, $\beta_{1}, \beta_{2} \ldots, n$ the partial regression coefficients, $X_{1}, X_{2} \ldots, X_{n}$ represent each of the independent variables.

In addition to the 230 rainfall-induced shallow landslide events, 230 random points that do not overlap with actual events are used to represent the absence of landslides as areas of "no-event yet". Buffers and thresholds are not applied to random points because statistically, these points have an equal probability of representing an event as much as a no-event. In this case, the pixel value is selected as a representative for random points.

The regression model calculations are performed using SPSS [34] statistical software. Various models are examined utilizing all threshold percentages, from where the best fitting threshold is selected. Likelihood-ratio for all variables is evaluated for removal when the contribution to the model is minimal. The contribution is deemed minimal if the observed significant level is greater than the probability 
of remaining in the model. In this study, such value is placed at the 0.05 level of significance.

\section{Results and Discussion}

Buffers and thresholds were designed to present a feasible approach to address misrepresentation of slope angle when monitoring landslide activity at high spatial resolution over a large domain. Improper identification of parameters, particularly for slope, often results in a misrepresentation of areas at risk. The development of this approach is only possible due to the availability of a comprehensive record of landslides events that represent the dominant characteristics of landslide-prone areas in the continental United States. A more extensive landslide record with the same characteristics is not available at the present moment, but using the buffer and threshold techniques in more data points can help minimize overestimation of susceptible areas at the large scale.

This work assumes that landslide risk is greater as slope angle increases. As the landslide inventory does not list the slope angle of the event because the locality is an estimate, slope values in each buffer area are tested. Comparison of slope percentile thresholds demonstrates that values below the $\mathrm{T}_{75}$ percentile threshold misrepresent areas of susceptibility by not including slope angle values higher than $35^{\circ}$, in this manner agreeing with previous studies $[6,26]$. Values below this threshold range between $0^{\circ}$ and $35^{\circ}$, this could result in susceptibility over estimations. In addition, this threshold does not account for higher slopes present in the area possibly resulting in a misrepresentation of reality as it is well known that landslides occur in a wider range of slope angles [7,15,35-37].

This same comparison demonstrates that values above the $\mathrm{T}_{95}$ threshold percentage encompass a wider range of slope values, but it is not clear whether these thresholds include over-estimations such as the inclusion of outliers. Therefore, the $\mathrm{T}_{99}$ threshold is investigated. Nevertheless, it is important to consider that threshold percentages above $\mathrm{T}_{95}$ could potentially represent better susceptible slope angle values, for this reason, each threshold is examined in a logistic regression analysis. Distribution for $\mathrm{T}_{\text {point }}, \mathrm{T}_{95}$, and $\mathrm{T}_{99}$ thresholds can be seen in Figure 4.

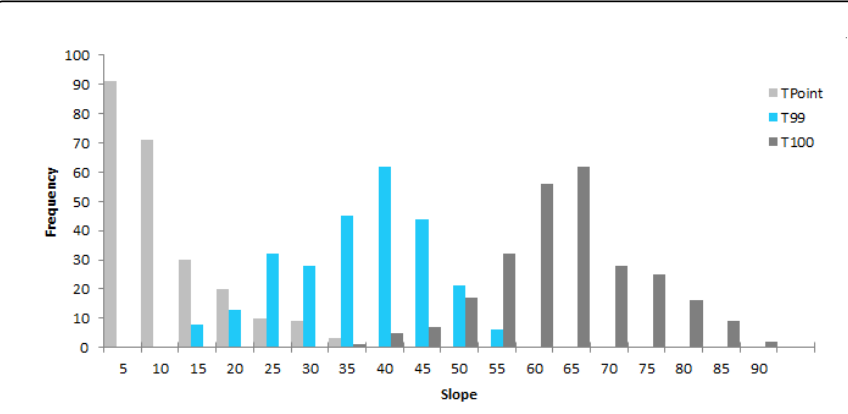

Figure 4: Slope angle distribution for threshold sensitivity analysis.

Further analysis of each threshold percentage is tested in a logistic regression model, it is determined that threshold $\mathrm{T}_{99}$ is the most suitable value because it produces the most representative range of slope values and it yields the best fitting model. In addition, results are consistent with local slope instability studies around the world [7,15,17,35-38]. Moreover, the highest amount of variation in the dependent variable is explained by the strongest relationship between the predictors and the prediction at $94.3 \%$. This slope threshold is a conservative assessment that no does not under or over-estimate slope angle susceptibility.

The performance of each model describes how well each variable describes the phenomenon as seen in Figure 5. Likelihood-ratio for all variables is evaluated for removal when the contribution is minimal. Contribution is deemed minimal if the observed significant level is greater than the probability of remaining in the model. In this study, elevation's contribution to the tested models was deemed insignificant; therefore elevation is excluded at this point from any further analysis.

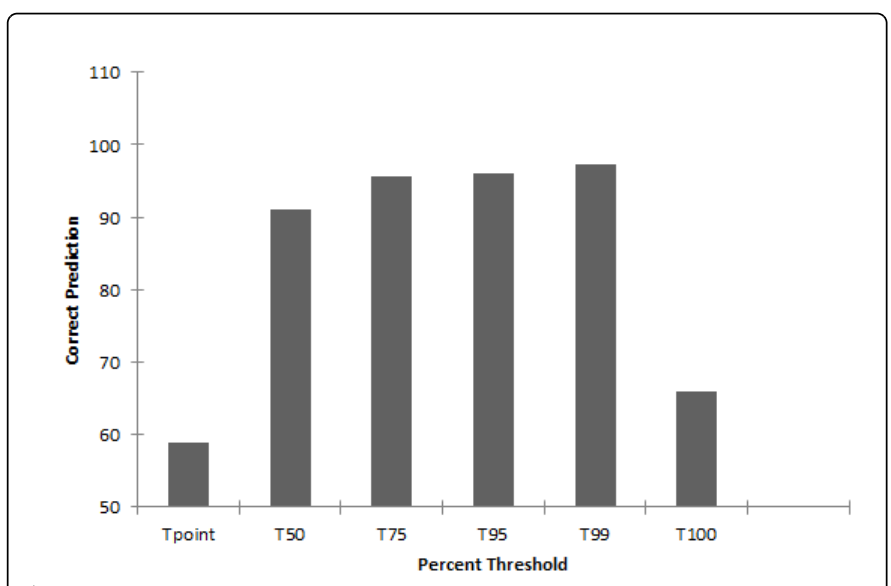

Figure 5: Percentage thresholds and their accuracy.

The independent variables in logistic regression can be characterized as useful predictors if the classification accuracy rate is substantially higher than the accuracy attainable by chance alone. SPSS calculates this chance accuracy criterion as the first step by not including any variables in the model. As a result, the accuracy rate computed for chance is $50.9 \%$ and the accuracy rate computed for the model is $97.2 \%$. This demonstrates that the variables included in the model significantly enhance the outcome. Table 2 shows the model's coefficients for each variable that is found to be significant. Slope and Land cover are significant variable predictors with $\mathrm{p}$-values $<0.01$ while soil type is less significant predictor with $\mathrm{p}$-value $<0.001$. Slope's significance as a predictor in the model, emphasizes the importance of its proper initial representation.

\begin{tabular}{|l|l|l|l|l|}
\hline Variable & B & S.E. & Wald & df \\
\hline Slope & 0.422 & 0.056 & 57.186 & 1 \\
\hline Soil Type & 0.009 & 0.046 & 0.042 & 1 \\
\hline Land Cover & -0.928 & 0.232 & 16.021 & 1 \\
\hline Constant & -5.012 & 1.549 & 10.464 & 1 \\
\hline
\end{tabular}

Table 2: Variables in the Equation.

As validation, the data was divided randomly on a $70-30 \%$ ratio for subsets as "model obtaining" and "validation" subsets respectively. Furthermore, the data was partitioned in $20 \%$ subsets for crossvalidation. Five rounds of cross-validation were performed using different partitions. Validation results represented by the average of the 
five rounds indicate that this model predicts the highest number of cases correctly at $97.2 \%$ accuracy.

A confusion matrix helps determine details about the performance of the model. The ability of the model to correctly identify the events is represented by True Positive. Events that are not correctly identified are represented by False Negative, and over predictions are represented by False Positive [33] (Table 3).

\begin{tabular}{|l|l|l|l|}
\hline \multicolumn{4}{|c|}{ Landslide Event } \\
\hline & Predicted & Not Predicted & Total \\
\hline Landslide & 220 true positive & 6 false positive & 226 \\
\hline Not landslide & 7 false negative & 227 true negative & 234 \\
\hline Total & 227 & 233 & 460 \\
\hline
\end{tabular}

Table 3: Confusion Matrix POD and FAR for all landslide events.

It is important to emphasize that this study only investigates the relationship of some static variables to landslide events. Rainfall, the triggering factor for the landslides in this study is not incorporated. It is assumed that by incorporating this factor in addition to other proxies like soil moisture into the logistic regression model will result in higher accuracy rate.

\section{Landslide probability mapping}

The logistic regression model is carried over to ArcGIS 10.2. All variables are processed based on their corresponding coefficients. It is important to reiterate that only static variables are used in this map and better resolution information

The resulting map in Figure 6 is classified into 2 categories based on a cut off value of 0.5: a) Not Probable (0-0.50: b) Probable (0.501-1).

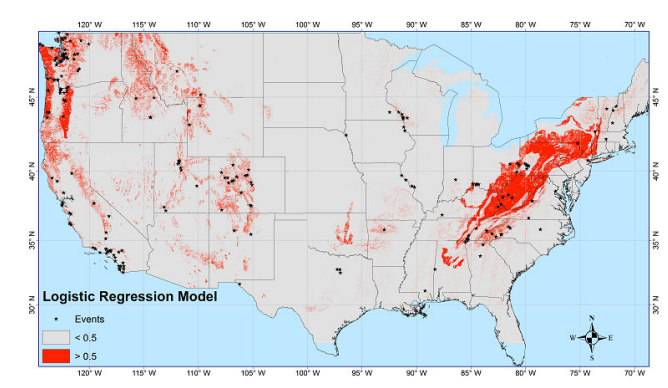

Figure 6: Landslide Probability.

\section{Conclusions}

Landslide studies at large scales are limited by uncertainties. At the present time, no system exists that can simultaneously address both regional and local scales. This work proposes utilizing buffer and threshold techniques to minimize uncertainty at the local scale so further analysis can be done on a larger scale. Various threshold percentages corresponding to 230 shallow landslides in the continental United States are tested logistic regression analysis. Findings are as follows:
Buffer analysis is efficient at narrowing large areas to more manageable scales. This, of course, depends on the original availability of a well-constructed landslide inventory that provides information on the event's locality.

Slope threshold percentage techniques confirm that slope susceptibility is misrepresented when performing analysis at large scales. Most slope values for thresholds lower than $\mathrm{T}_{75}$ do not include slopes higher than 350 , this result in the overestimation of susceptible areas and a misrepresentation of reality as landslide-prone slopes has a greater range.

It is determined that the threshold percentage $\mathrm{T}_{99}$ is a conservative assessment that includes a wider range of slope angles and successfully excludes outliers.

A regional logistic regression model demonstrates that utilizing the threshold percentage $\mathrm{T}_{99}$ to model slope instability at large scales results in an accuracy rate of $97.2 \%$.

Likelihood-ratio for all variables is evaluated in the logistic model, elevation's contribution was deemed insignificant therefore excluded from the model.

Using the buffer and threshold techniques in more data points can help minimize overestimation of susceptible areas at the large scale.

Eliminating uncertainties at the local level improves the large scale modeling accuracy.

It is important to note that given the restrictions of physically or insitu base data, this study is subject to the existence of a comprehensive landslide inventory and reasonably scaled surface data. Better resolution information and other static parameters can be tested in logistic regression analysis, but awareness of limitations given the large scale is imperative as some data may be deemed too general and not sufficiently detailed for the mapping scale. In addition, although the focus of this study is rain-triggered shallow landslides, neither rain nor antecedent soil moisture information has been implemented in this work. It is assumed that future implementation of these unaccounted for variables and the addition of more detailed soil information (in the continental U.S.) will help describe susceptibility conditions dynamically, therefore, enhancing this platform. Moreover, it is possible for this approach to be brought to other regions, as all the data used in the present analysis is available globally. The approach should be regional, leading to a global scale in order to minimize over generalizations.

\section{Acknowledgements}

This publication was made possible by the National Oceanic and Atmospheric Administration, Office of Education Educational Partnership Program award NA11SEC4810004. Its contents are solely the responsibility of the award recipient and do not necessarily represent the official views of the US Department of Commerce, National Oceanic and Atmospheric Administration.

\section{References}

1. Petley D (2012) Global patterns of loss of life from landslides. Geol 40: 927-930.

2. http://landslides.usgs.gov/learn/ls101.php

3. Brocca L, Ponziani F, Moramarco T, Melone F, Berni N, et al. (2012) Improving Landslide Forecasting Using ASCAT-Derived Soil Moisture 
Data: A Case Study of the Torgiovannetto Landslide in Central Italy. Remote Sens 4: 1232-1244.

4. Cruden DJ, Varnes DM (1996) Landslides: Investigation and Mitigation. Transp Res board Spec Rep 247: 36-75.

5. Hong YHY, Adler RF, Huffman G (2007) An Experimental Global Prediction System for Rainfall-Triggered Landslides Using Satellite Remote Sensing and Geospatial Datasets. IEEE Trans Geosci Remote Sens 45: 1671-1680.

6. Kirschbaum DB, Adler R, Hong, Lerner-Lam (2009) Evaluation of a preliminary satellite-based landslide hazard algorithm using global landslide inventories. Nat Hazards Earth Syst Sci 9: 673-686.

7. van Westen CJ, Castellanos E, Kuriakose SL (2008) Spatial data for landslide susceptibility, hazard, and vulnerability assessment: An overview. Eng Geol 102: 112-131.

8. Abe K, Ziemer RR (1991) Effect of Tree Roots on Shallow-Seated Landslides.

9. Schmidt KM, Roering JJ, Stock JD, Dietrich WE, Montgomery DR, et al. (2001) The variability of root cohesion as an influence on shallow landslide susceptibility in the Oregon Coast Range. Can Geotech J 38: 995-1024.

10. Kitutu M, Muwanga A (2009) Influence of soil properties on landslide occurrences in Bududa district, Eastern Uganda. African J 4: 611-620.

11. Aristizábal E, García E, Martínez C (2015) Susceptibility assessment of shallow landslides triggered by rainfall in tropical basins and mountainous terrains. Nat Hazards 78: 621-634.

12. Eeckhaut M, Hervás J, Jaedicke C, Malet JP, Montanarella L, et al. (2011) Statistical modelling of Europe-wide landslide susceptibility using limited landslide inventory data. Landslides 9: 357-369.

13. Fell R, Corominas J, Bonnard C, Cascini L, Leroi E, et al. (2008) Guidelines for landslide susceptibility, hazard and risk zoning for landuse planning. Eng Geol 102: 99-111.

14. Ghosh S, Westen CJ, Carranza EJM, Jetten VG (2011) Integrating spatial, temporal, and magnitude probabilities for medium-scale landslide risk analysis in Darjeeling Himalayas, India. Landslides 9: 371-384.

15. Pareta K, Pareta U (2012) Landslide Modeling and Susceptibility Mapping of Giri River Watershed, Himachal Pradesh (India). Int J Sci 1: 91-104.

16. Teerarungsigul S (2006) Landslide Prediction Model Using Remote Sensing GIS and Field Geology: A Case Study of Wang Chin District, Phrae Province, Conf. Geol.

17. Dai F, Lee C (2002) Landslide characteristics and slope instability modeling using GIS, Lantau Island, Hong Kong. Geomorphology 42: 213-228.

18. Liao Z, Hong Y, Wang J, Fukuoka H, Sassa K, et al. (2010) Prototyping an experimental early warning system for rainfall-induced landslides in Indonesia using satellite remote sensing and geospatial datasets. Landslides 7: 317-324.

19. Kirschbaum DB, Adler R, Hong Y (2012) Advances in landslide now casting: evaluation of a global and regional modeling approach. Environ Earth, pp: 1683-1696.

20. Bui DT, Pradhan B, Lofman O, Revhaug I, Dick OB (2012) Landslide susceptibility assessment in the Hoa Binh province of Vietnam: A comparison of the Levenberg-Marquardt and Bayesian regularized neural networks. Geomorphology 171-172: 12-29.
21. Glade T, Crozier M, Smith P (2000) Applying probability determination to refine landslide-triggering rainfall thresholds using an empirical Antecedent Daily Rainfall Model. Pure Appl Geophys 157: 1059-1079.

22. Nefeslioglu HA, Gokceoglu C, Sonmez H (2008) An assessment on the use of logistic regression and artificial neural networks with different sampling strategies for the preparation of landslide susceptibility maps. Eng Geol 97: 171-191.

23. Pradhan B (2010) Manifestation of an advanced fuzzy logic model coupled with Geo-information techniques to landslide susceptibility mapping and their comparison with logistic regression modelling. Environ Ecol Stat 18: 471-493.

24. Jaedicke C, Van Den Eeckhaut M, Nadim F, Hervas J, Kalsnes B, et al. (2013) Identification of landslide hazard and risk 'hotspots' in Europe, Bull. Eng. Geol. Environ 73: 325-339.

25. Nourani V, Pradhan B, Ghaffari H, Sharifi SS (2013) Landslide susceptibility mapping at Zonouz Plain, Iran using genetic programming and comparison with frequency ratio, logistic regression, and artificial neural network models. Nat Hazards 71: 523-547.

26. Nadim F, Kjekstad O, Peduzzi P, Herold C, Jaedicke C (2006) Global landslide and avalanche hotspots, Landslides 3: 159-173.

27. Ayalew L, Yamagishi H (2005) The application of GIS-based logistic regression for landslide susceptibility mapping in the Kakuda-Yahiko Mountains, Central Japan. Geomorphology 65: 15-31.

28. Radbruch-Hall D, Colton R, Davies W, Luccitta I, Skipp B, et al. (1982) Landslide overview map of the conterminous United States, US, Geol Surv.

29. Zhu L, Huang J (2006) GIS-based logistic regression method for landslide susceptibility mapping in regional scale. J Zhejiang Univ Sci A 7: 2007-2017.

30. Land G, Share C, Latham J, Cumani R, Rosati I, et al. (2014) Global Land Cover SHARE.

31. http://landslides.usgs.gov/recent/index.php?year=2014

32. Aristizábal E, Velez J, Martínez C, Jaboyedoff M (2015) SHIA_Landslide: a distributed conceptual and physically based model to forecast the temporal and spatial occurrence of shallow landslides triggered by rainfall in tropical and mountainous basins, Landslides.

33. Triola M (2013) Elementary Statistics, Tenth Pearson.

34. IBM (2013) SPSS. Armonk, NY.

35. Wu X, Niu R, Ren F, Peng L (2013) Landslide susceptibility mapping using rough sets and back-propagation neural networks in the Three Gorges, China, Environ. Earth Sci 70: 1307-1318.

36. Pourghasemi HR, Moradi HR, Aghda SMF (2013) Landslide susceptibility mapping by binary logistic regression, analytical hierarchy process, and statistical index models and assessment of their performances. Nat Hazards 69: 749-779.

37. Chen HX, Zhang LM (2014) A physically-based distributed cell model for predicting regional rainfall-induced shallow slope failures. Eng Geol 176: 79-92.

38. The ASTER product was obtained courtesy of the NASA EOSDIS Land Processes Distributed Active Archive Center (LP DAAC), USGS/Earth Resources Observation and Science (EROS) Center, Sioux Falls, South Dakota (lpdaac.usgs.gov, 2015). 